\title{
The Cosmic-Ray-Induced Radiation Environment and Dose to Man for Low-Orbit Space Applications
}

\author{
H. A. Sendmeier \\ G. E. Hansen \\ M. E. Battat* \\ K. O'Brien**
}

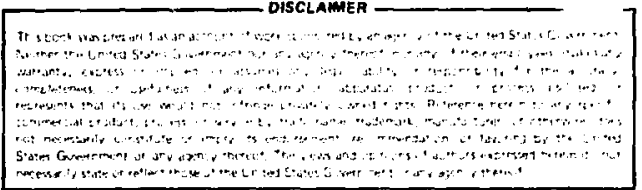

"Consultant. 1817 Shelwood Blvd., NE, Albuquerque, NM 87112.

" Department of Energy. Environmental Measurement Laboratory, New York, NY 10014.

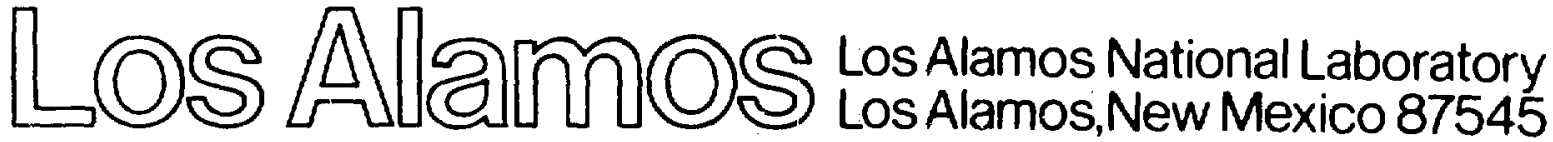


THE COSMIC-RAY INDUCED RADIATION ENVIRONMENT

AND DOSE TO MAN FOR LOW-JSBIT SPACE APPLICATIONS

by

H. A. Sandmeier, G. E. Hansen, M. E. Battat, and K. O'Brien

\section{ABSTRACT}

Neutrons and photons resuiting from the interaction of galactic cosmic rays with the material of an orbiting satellite or an orbiting space station at an altitude of some few hundreds of kilometers, and below the level of the radiation belts, rave been calcuiated as a function of geomagnetic latitude and solar activity level. The photon and neutron leakage currents from the top of the atmosphere have been computed. The radiation dose-equivalent rate to an unshielded astronaut has also been calculated. The maximum doseequivalent rate, near the magnetic poles, was $2 \mathrm{mrem} / \mathrm{h}$. In deep space this would amount to $18 \mathrm{rem} / \mathrm{yr}$, indicating that for a prolonged stay in space, shielding would be needed.

\section{INTRODUCTION}

In a previous publication by the authors, 1 data were presented for the cosmic-ray induced neutron sources as a function of depth in several materials. Using this source information, neutron fluxes and spectra were calculated for air/ground, air/seawater, air/iron, and air/aluminum geometries. Good agreement with experimental data was obtained at the air/ground interface. Subsequently, we applied our calculational techniques to compute the neutron and gamma-ray fluxes and spectra in the vicinity of an aircraft at selected altitudes in the atmosphere.

The purpose of this report is to characterize the radiation environment of interest for low-orbit space applications. By low orbit we mean an altitude of a few hundred kilometers above sea level, but below any radiation belts. The starting point for calculations of this type is a knowledge of the primary cosmic-ray flux and its interaction with the atmosphere and objects in space. Because of its complexity, details as to the particle composition and energy 
spectra of the primary radiation will not be discussed here. However, we have included in Appendix $A$ a list of the nuclear reactions that take place as cosmic-rays propagate through the atmosphere.

For this study, we have evaluated numerically the following components of the radiation field at a few hundred kilometers above sea level.

- Neutron and gamma-ray leakages from top of atmosphere due to cosmicray induced neutrons

- Gamma-ray leakage from top of atmosphere due to interaction of primary cosmic-ray flux with the atmosphere

- Diffuse cosmic gamma-ray radiation at top of atmosphere

- Neutron and gamma-ray leakages from surface of satellite or space station due to cosmic-ray induced neutrons

- Gamma-ray leakages from surface of satellite due to primary cosmicray neutrons and protons

- Cosmic-ray induced whole-body dose to an astronaut.

The effects of varying geomagnetic latitude and solar activity were investigated for the first component listed above. For the other components, average solar conditions were assumed; latitude effects were examined in varying degrees of detai1. Except for the diffuse cosmic radiation, the above components are illustrated schematically in Fig. 1.

Although not of direct interest to the subject matter of this report, results of calculations to determine the neutron and gamma-ray fluxes in the atmosphere are summarized in Appendix $C$. Only those interactions between cosmicray induced neutrons and the atmosphere were considered. Calculations were made for latitudes of $30-40^{\circ}$ and $50-60^{\circ}$ and solar activity conditions corresponding to Ehmert potentials of 200 and $1000 \mathrm{MV}$.

11. COSMIC-RAY INOUCED NEUTRON AND GAMMA-RAY LEAKAGES AND SPECTRA FROM TOP OF ATMOSPHERE AS A FUNCTION OF LATITUDE AND SOLAR ACTIVITY

The primary cosmic radiation incident on the earth's atmosphere is composed of protons $(87 \%)$, alpha particles $(11 \%)$, heavier nuclei $(\sim 1 \%)$, and electrons ( - 1\%). These particles interact with air nuclei and produce secondary neutrons and protons, as well as charged and uncharged pions. It is the transport of these secondary neutrons in the atmosphere that we consider in this section. As shown in Fig. 1 of reference 1, the neutron source per unit mass of air decreases exponentially as one goes down in the atmosphere. Figure 3 of reference 1 shows the energy spectrum of these neutrons; the energy ranges from 0.1 to $100 \mathrm{MeV}$, with a peak at $10 \mathrm{MeV}$. Using the latter source specifications, and taking the atmosphere to be a semi-infinite slab, we then solved the multigroup transport equation. Because the top group in our multigroup cross-section set is in the 15.73- to 17.0-MeV range, neutrons above $15.73 \mathrm{MeV}$ were assigned to the top group. In their transport through the atmosphere, the neutrons produce 


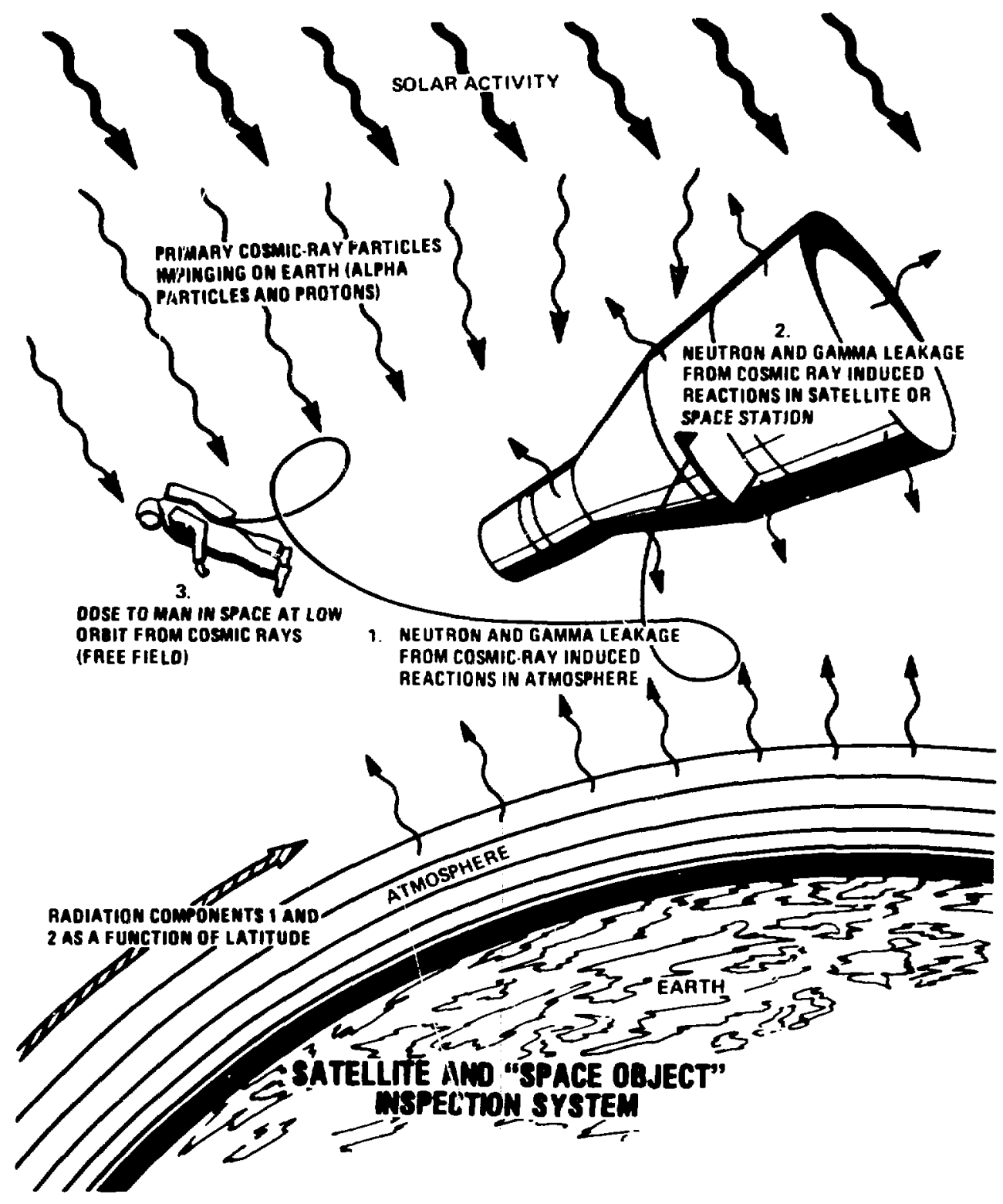

Fig. 1. Componerts of the radiation environment encountered in low-orbit space trajectories. 
secondary photons, mainly as a result of inelastic and capture processes. Results of transport calculations yield, among other things, the flux distribution (space and energy) of neutrons and photons in the atmosphere and also the ieakage currents of these particles from the top of the atmosphere. These leakage currents are one of the components of the radiation field at altitudes corresponding to low-orbit space trajectories.

At this point we digress to clarify what is meant by the term "top of the atmosphere." There is no definite upper limit or top to the atmosphere because at great heights it blends imperceptibly into the interplanetary medium. However, since the density distribution of the upper atmosphere is exponential, there is a finite mass of air overhead at any altitude and we have chosen to use this mass (in terms of grams of air per square centimeter of the earth's surface) as our dimension of depth. Introducing these coordinates of mass per unit area (or "areal density") into the one-dimensional slab-geometry Boltzmann equation is both easy and natural, making it simple to describe the atmosphere as a slab of finite thickness for purposes of calculation. Of course, the final results can be expressed in terms of altitude if the correct conversion between altitude and atmospheric depth is applied.

The transport calculations were performed uşing the ONETRAN code (see Appendix B) with an atmospheric thickness of $240 \mathrm{~g} / \mathrm{cm}^{2}$. The total thickness of the atmosphere - $1033 \mathrm{~g} / \mathrm{cm}^{2}$ at sea level - was not used because caiculations showed that the top leakage did not change significantly as the thickness was increased beyond $240 \mathrm{~g} / \mathrm{cm}^{2}$. Several problems were run to assess the effects of changes in latitude and Ehmert potentials. The Ehmert potential, expressed on a scale between 0 and 1000 megavolts (MV), is a measure of the solar activity (or solar modulation). Solar activity waxes and wanes with an approximate 11-year cycle. An increase in tie Ehmert potential corresponds to a decline in the cosmic-ray intensity, while a decrease causes a corresponding rise. At the time of writing, the solar activity is nearing its maximum (approximately $700 \mathrm{MV}$ ) and the corresponding intensities are near their minimum.

Results of these calculations are plotted in Fig. 2 and summarized in Table I. At a given geomagnetic latitude, the leakages increase as the Ehmert potential is decreased, and the change with potential is more pronounced at the higher latitudes. It is also seen that the maximum leakage current is about $4 / \mathrm{cm}^{2}-\mathrm{s}$. In Table II we list information of the spectra of neutrons and photons at a latitude of 30-40 degrees and an Ehmert potential of $200 \mathrm{MV}$. The latter value corresponds to average solar activity conditions, averaged over the last 200 years. It is observed that half of the leakage neutrons are above $3 \mathrm{MeV}$ and that 70 percent of the photons have energies below $0.5 \mathrm{MeV}$.

III. COSMIC-RAY INDUCED (DIRECT PRODUCTION) GAMMA-RAY LEAKAGES AND SPECTRA FROM TOP OF ATMOSPHERE AT 35 AND 65 DEGREES LATITUDE; AVERAGE SOLAR ACTIVITY

In addition to the neutron-induced gamma rays (section II), low-energy photons are produced by bremsstrahlung from secondary cascade electrons. As noted in Appendix $A$, the reactions responsible for this electron production are principally (1) the decay of charged muons and (2) the decay of neutral pions followed by pair production processes. In the first stage of its decay, the 


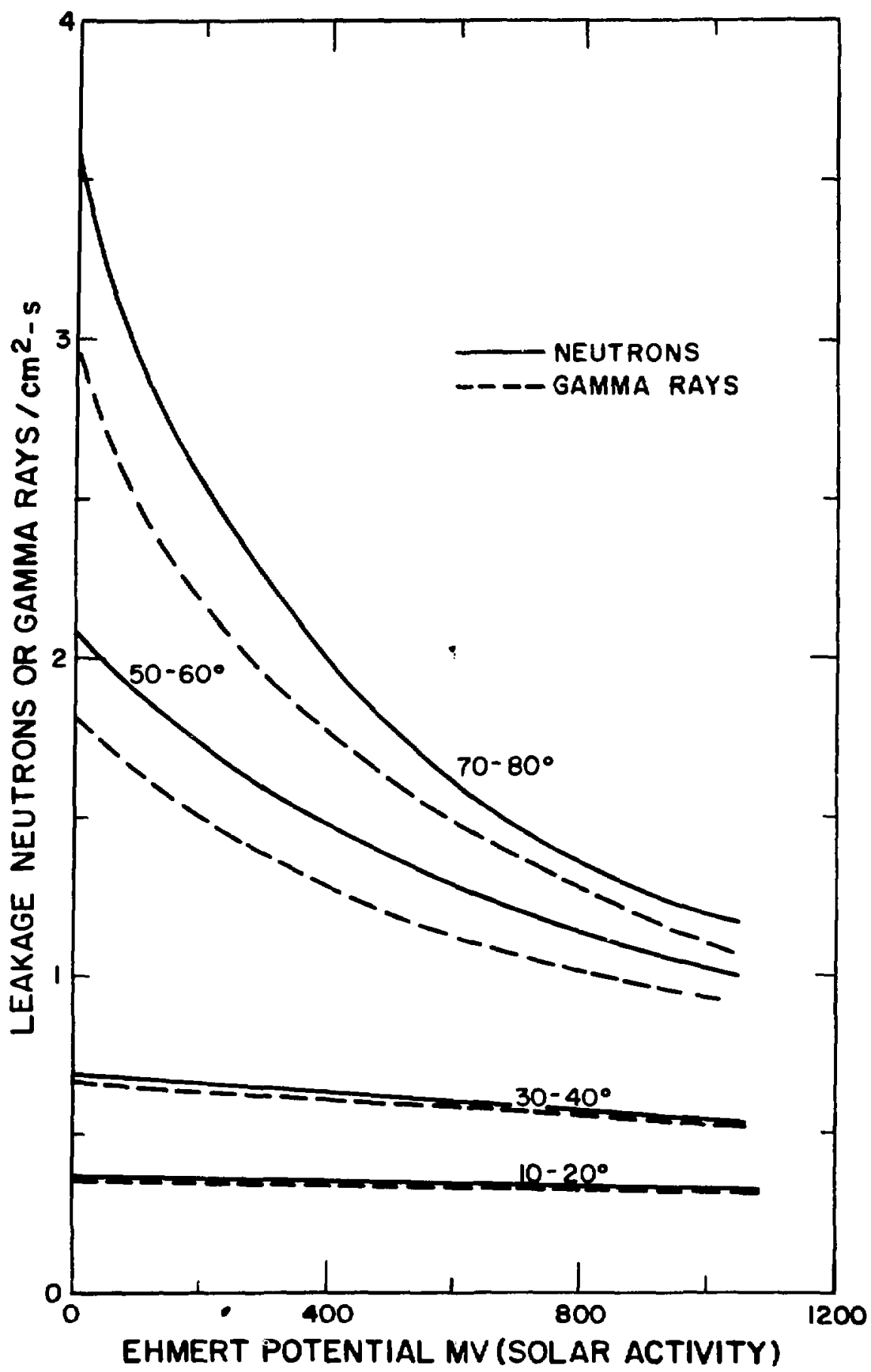

Fig. 2. Neutron and gamma-ray leakages from top of atmosphere as a function of latitude and Ehmert potential. 
TABLE I

\begin{tabular}{|c|c|c|c|c|c|c|c|c|}
\hline \multirow{3}{*}{$\begin{array}{l}\text { DLGREES } \\
\text { LATITUOE }\end{array}$} & \multicolumn{8}{|c|}{ EHMENT POTENTIAL IMVI } \\
\hline & \multicolumn{2}{|c|}{0} & \multicolumn{2}{|c|}{200} & \multicolumn{2}{|c|}{500} & \multicolumn{2}{|c|}{1000} \\
\hline & $N$ & $\mathbf{G}$ & $\mathbf{N}$ & $\mathbf{G}$ & $\mathbf{N}$ & $\mathbf{G}$ & N & $\mathbf{G}$ \\
\hline $0-10$ & 0.36 & 0.35 & 0.34 & 0.34 & 0.33 & $\overline{0.33}$ & 0.31 & 0.31 \\
\hline $10-20$ & 0.37 & 0.35 & 0.39 & 0.38 & 0.35 & 0.34 & 0.33 & 0.32 \\
\hline $20-30$ & 0.52 & 0.51 & 0.50 & 0.40 & 0.48 & 0.40 & 0.44 & 0.43 \\
\hline $30-\infty$ & o.es & o.es & o.es & 0.63 & 0.62 & 0.90 & 0.55 & 0.53 \\
\hline $40-50$ & 1.1 & o.es & 0.6 & 0.81 & 0.88 & 0.12 & 0.74 & 0.69 \\
\hline $50-\infty$ & 2.1 & 1.0 & 1.7 & 1.5 & 1.4 & .1 .2 & 1.0 & 0.94 \\
\hline $60-70$ & 3.4 & 2.0 & 2.5 & 2.1 & 1.0 & 1.5 & 1.2 & 1.1 \\
\hline 20 - & 3.6 & 3.0 & 2.6 & 2.2 & 1.8 & 1.0 & 1.2 & 1.1 \\
\hline
\end{tabular}

TABLE II

PFETRUM OF SECONDARY NEUTRONS ANO GAMMA-RAYS LEAKING EROM TOP OF ATMOSPHERE. LATITUDE - 30-40 DEGAEES. AVEAAGE SOLAR ACTIVITY.

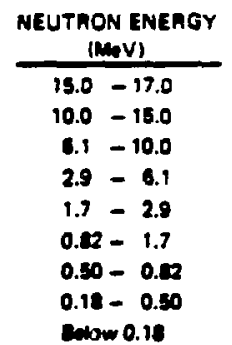

\begin{tabular}{c}
$\begin{array}{c}\text { GAMMA.RAY ENERGY } \\
\text { (REV) }\end{array}$ \\
\hline $4.0-10.0$ \\
$3.0-4.0$ \\
$2.0-3.0$ \\
$1.2-2.0$ \\
$1.0-1.2$ \\
$0.1-1.0$ \\
$0.5-0.8$ \\
$0.01-0.3$
\end{tabular}

PERCENT OF NEUTAON LEAKAGE GURRENT

\begin{tabular}{c} 
PERCENT OF GAMMA-AAY \\
LEAKAGE CURRENT \\
\hline 6.0 \\
3.2 \\
3.6 \\
4.0 \\
3.3 \\
2.4 \\
6.1 \\
72.4
\end{tabular}


neutral pion decays to two photons, each with energy of approximately $70 \mathrm{MeV}$. High-energy photons are also present in the atmosphere because, in bremsstrahlung, the photon spectrum covers the range from zero up to the kinetic energy of the electron.

Calculation of the leakage of gamma rays produced by the interaction of primary cosmic-rays with the atmosphere were perfurmed using a new Monte Carlo version of the HASL/KO'B cosmic-ray interaction code (Appendix A). Note that, as in Section II, it is the leakage from the top of the atmosphere that is of interest rather than the flux distribution in the atmosphere. In order to minimize the number of calculations, we have examined two cases only, 35 and 65 degrees latitude; average solar activity conditions (200 MV Ehmert potential) were assumed. Results of the calculations are listed in Table III. Comparing these results with those in Section II, we observe that the photon leakages from direct production are a factor of 6 to 10 less than for the neutron-induced gamma rays. The photon energy bins in Table III are much coarser than the ones provided by the code. Except for the top two energy groups, the spectra at the two latitudes are somelshat similar; in both cases, 70 to 75 percent of the photons are below 0.5 MeV. At 35 degrees latitude, the spectrum is also similar to that shown in Table II - 75 percent of the photons are below $0.5 \mathrm{MeV}$ for both components.

\section{TABLE III}

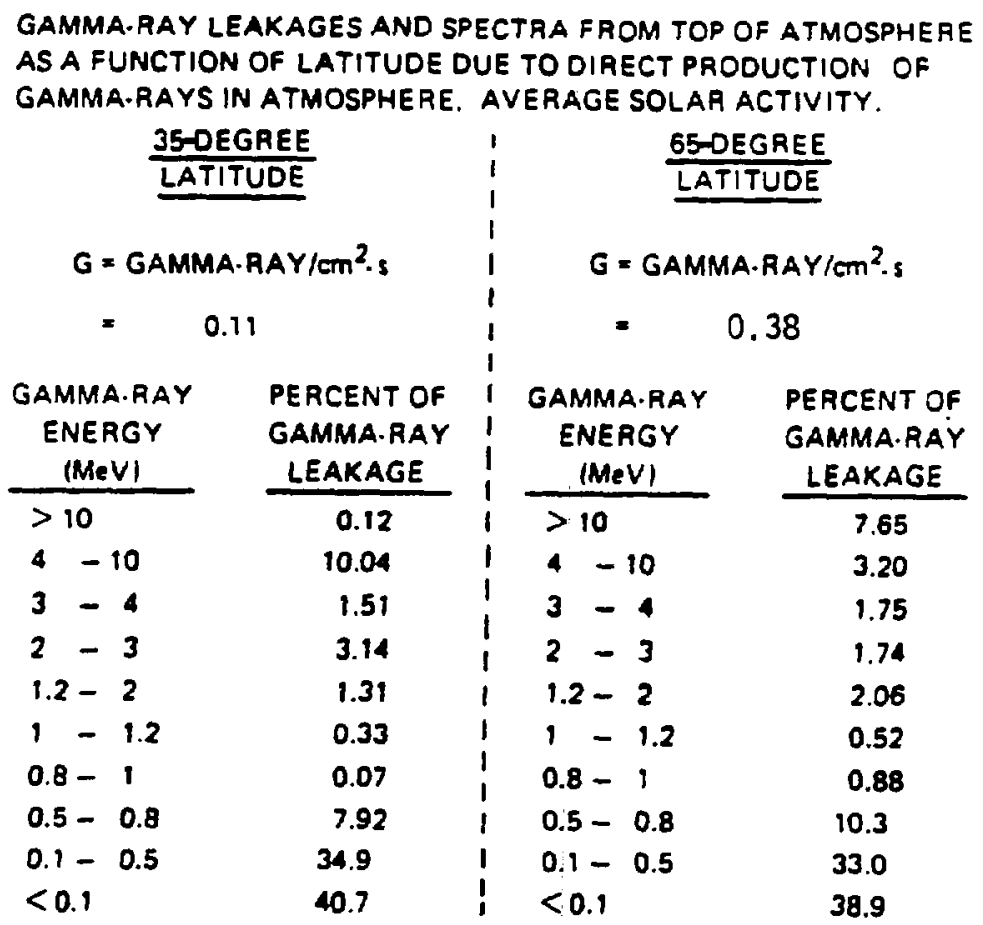




\section{DIFFUSE COSMIC GAMMA RADIATION}

The subject of oiffuse cosmic $x$ - and gamma-radiation is a relatively new branch of astrophysics and much effort has been expended to understand and characterize this radiation. Measurements of gamma radiation in the upper atmosphere ${ }^{3}$ indicate the presence of a diffuse extragalactic component superposed on the "local" or atmospheric gamma rays (see sections II and III). This diffuse flux is maximum at the top of the atmosphere, decreasing to well below the atmospheric component at depths of a few tens of grams per square centimeter. The mechanisms that give rişe to an extragalar:tic diffuse gamma-ray flux are not as yet clearly understood.

In the energy range from 0.1 to $100 \mathrm{MeV}$, the results of many measurements ${ }^{3}$ are consistent with a spectrum of the form

$$
0.011 \mathrm{E}^{-2.3} \text { photons } / \mathrm{cm}^{2}-\mathrm{s}-\mathrm{sr}-\mathrm{MeV} \text {, }
$$

where $E$ is the photon energy in million electron volts. The spectrum of this background flux extends into the few kiloelectron volt region. For example, fluxes of $7.9 \pm 3$ photons $/ \mathrm{cm}^{2}-5-s r$ have been reported in the $1-$ to $12-\mathrm{keV}$ region. If the above expression is integrated from 0.1 to $10 \mathrm{MeV}$, the computed flux is 0.163 photons $/ \mathrm{cm}^{-s-s r}$. Multiplying by $2 \pi \mathrm{sglid}$ angle - the region of the sky above the horizon - we obtain 1.1 photons $/ \mathrm{cm}^{2}-5$ for the diffuse gamma-ray flux in the range from 0.1 to $10 \mathrm{MeV}$. It is noted that $88 \%$ of the flux is in the $0.1-$ to $0.5-\mathrm{MeV}$ range.

V. COSMIC-RAY INDUCED NEUTRON AND GAMMA-RAY LEAKAGE AND SPECTRA FROM SURFACE OF A SATELLITE AS A FUNCTION OF LATITUDE; AVERAGE SOLAR ACTIVITY

For these ONETRAN calculations (see Appendix $B$ ), we represented the satellite as an aluminum sphere of $1.9-\mathrm{m}(6.2-\mathrm{ft})$ radius and average density $(\rho)$ of $0.0175 \mathrm{~g} / \mathrm{cm}^{3}$. This translates to a $\rho R$ of $3.3 \mathrm{~g} / \mathrm{cm}^{2} \mathrm{Al}$ and a weight of $505 \mathrm{~kg}$ (1100 Ibs). As in Section II, the source was constructed by combining information on stars/g-s in Al, density of Al, and neutrons/star. An example of the neytron spectrum in $A 1$ is shown in Fig. 3 of reference 1 . Because a $\rho R$ of $3.3 \mathrm{~g} / \mathrm{cm}^{2}$ corresponds to a thickness of $1.2 \mathrm{~cm}$ of normal-density $\mathrm{Al}$, the source intensity in the satellite was taken to be constant. In addition to surface leakages, we also obtained fluxes in a vacuum region outside the satellite for radii up to 300 meters (approximately $1000 \mathrm{ft}$ ). The flux information is voluminous and will not be discussed here, but the information is available. To a good approximation $( \pm 10 \%)$, the fluxes for radii greater than 20 meters $(966 \mathrm{ft})$ can be estimated by assuming the particles leaking from the satellite surface to be concentrated at a point at the center of the sphere. An Ehmert potential of 200 MV (average solar activity) was assumed for the calculations.

Results of the calculations are plotted in Fig. 3 and summarized in Table IV. Note that we show the total leakage from the satellite surface - hence the large numbers. Expressed in terms of surface currents, the neutrons $/ \mathrm{cm}^{2}-\mathrm{s}$ values vary between 0.013 and 0.35 ; the photon currents are in the range from $8.9 \times 10^{-4}$ to 0.024 . The neutron leakage of $10^{4}$ to $10^{5} \mathrm{n} / \mathrm{s}$ is comparable to that from a few kilograms of $\mathrm{Pu}$. In Table $V$ we show the leakage spectra at a latitude of 35 degrees. Compared to the leakage spectra from the top of the atmosphere 


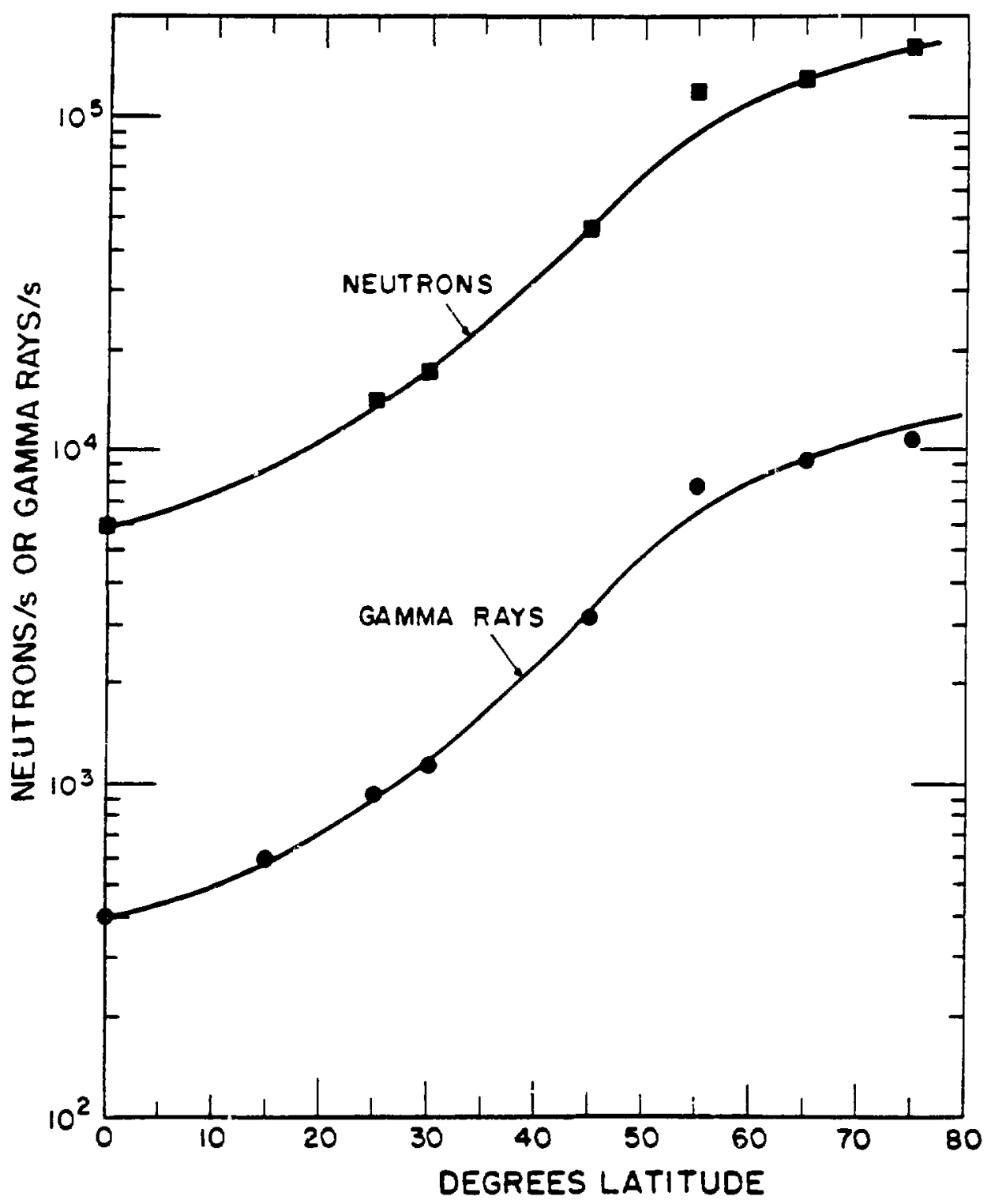

Fig. 3. Cosmic-ray induced neutron and gamma-ray leakages from satellite surface as a function of latitude. Average solar activity. 


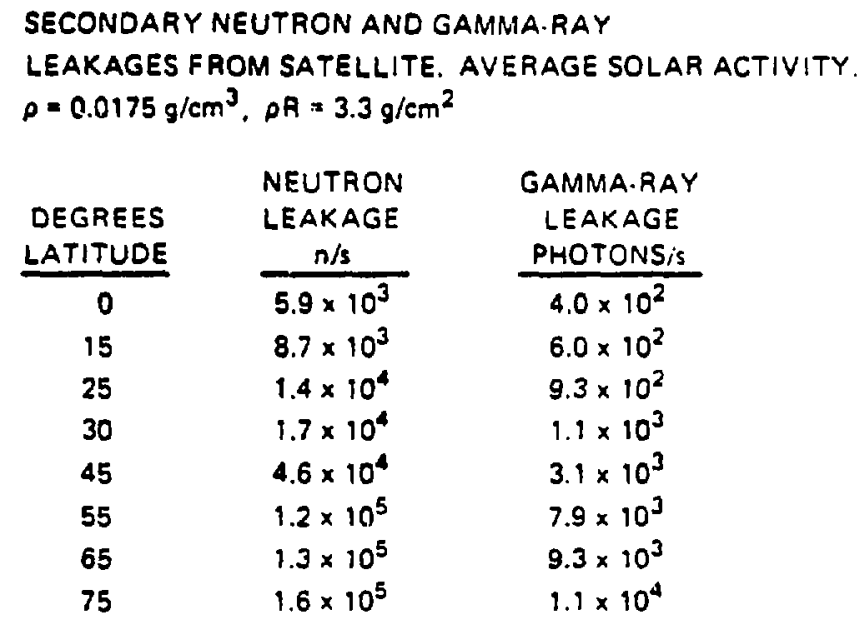

(Section III), these spectra are much harder; half of the neutrons are above $12 \mathrm{MeV}$, and 55\% of the photons have energies above $1 \mathrm{MeV}$.

VI. GAMMA-RAY LEAKAGE FROM SATELLITE DUE TO PRIMARY COSMIC-RAY FLUX (DIRECT PRODUCTION) AS A FUNCTION OF LATITUDE; AVERAGE SOLAR ACTIVITY

Calculations have been made, for conditions of average solar activity, to assess the magnitude of the photon leakage from a satellite, due to interactions of the primary cosmic-ray flux with the $A 1$ structure assumed. To be consistent with the model used in Section $V$ (cosmic-ray induced neutrons), a slab of Al with an areal density of $3.3 \mathrm{~g} / \mathrm{cm}^{2}$ was specified. Leakages were computed for the top and bottom surfaces of the slab and the contributions from primary protons and neutrons* were computed separately. In Figs. 4 and 5 are plotted the leakage currents for the latter two cases. First, we see that the photon leakage resulting from proton interactions is about a factor of 10 higher than the neutron contribution. Also, the bottom leakage is somewhat higher than the top leakage in all cases. Insofar as the photon leakage current is concerned, the primary cosmic-ray protons yield values between 0.01 and 0.1 as compared to $10^{-3}$ to 0.02 (Section V) for the secondary gamma rays. As for the photon spectra, tabulated in Table VI, we observe that the photon energies are much higher than calculated heretofore. For the proton-induced photons leaking out the top, $25 \%$ are between

*The primary cosmic-ray flux consists of energetic protons, alpha particles, and a small admixture of heavier nuclei that are omitted in this treatment. In interacting with materials, the alpha particles break up to form neutrons and protons. 


\section{TABLE V}

\section{SECONDARY NEUTRGN AND GAMMA.RAY LEAKAGE}

SPECTRA FOR SATELLITE. LATITUOE = 35 DEGREES.

\begin{tabular}{l} 
NEUT AON ENERGY \\
(MEV) \\
\hline
\end{tabular}

$15.0-17.0$

$12.0-15.0$

$7.8-12.0$

$3.7-7.8$

$2.2-3.7$

$1.4-2.2$

$0.50-9.4$

$018-0.50$

Below 0.18

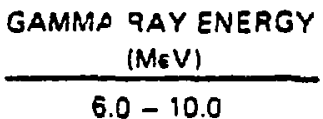

$4.0-0.0$

$3.0-4.0$

$2.0-3.0$

$1.2-2.0$

$1.0-1.2$

$0.8-1.0$

$0.5-0.8$

$0.01-0.5$
PERCENT OF NEUTRON

LËAKAGE

40.4

11.0

18.0

19.0

5.7

3.0

2.3

0.5

0.1

PERCENT OF GAMMA.RAY

LEAKAGE

\section{2}

5.0

4.7

6.1

14.1

21.9

27.2

10.8

7.0

4 and $100 \mathrm{MeV}$; the spectrum for the bottom leakage is somewhat similar. The spectra for the neutron-induced photon leakage shows a definite difference in the top and bottom spectra. The photons leaking out the top are predominantily (75\%) below $2 \mathrm{MeV}$, but 60\% of the bottom leakage is due to photons above $4 \mathrm{MeV}$. In comparing the neutron-induced (Section $V$ ) and direct-production photon leakages, the relative importance of number flux and spectra will depend on the particular response function being considered. 


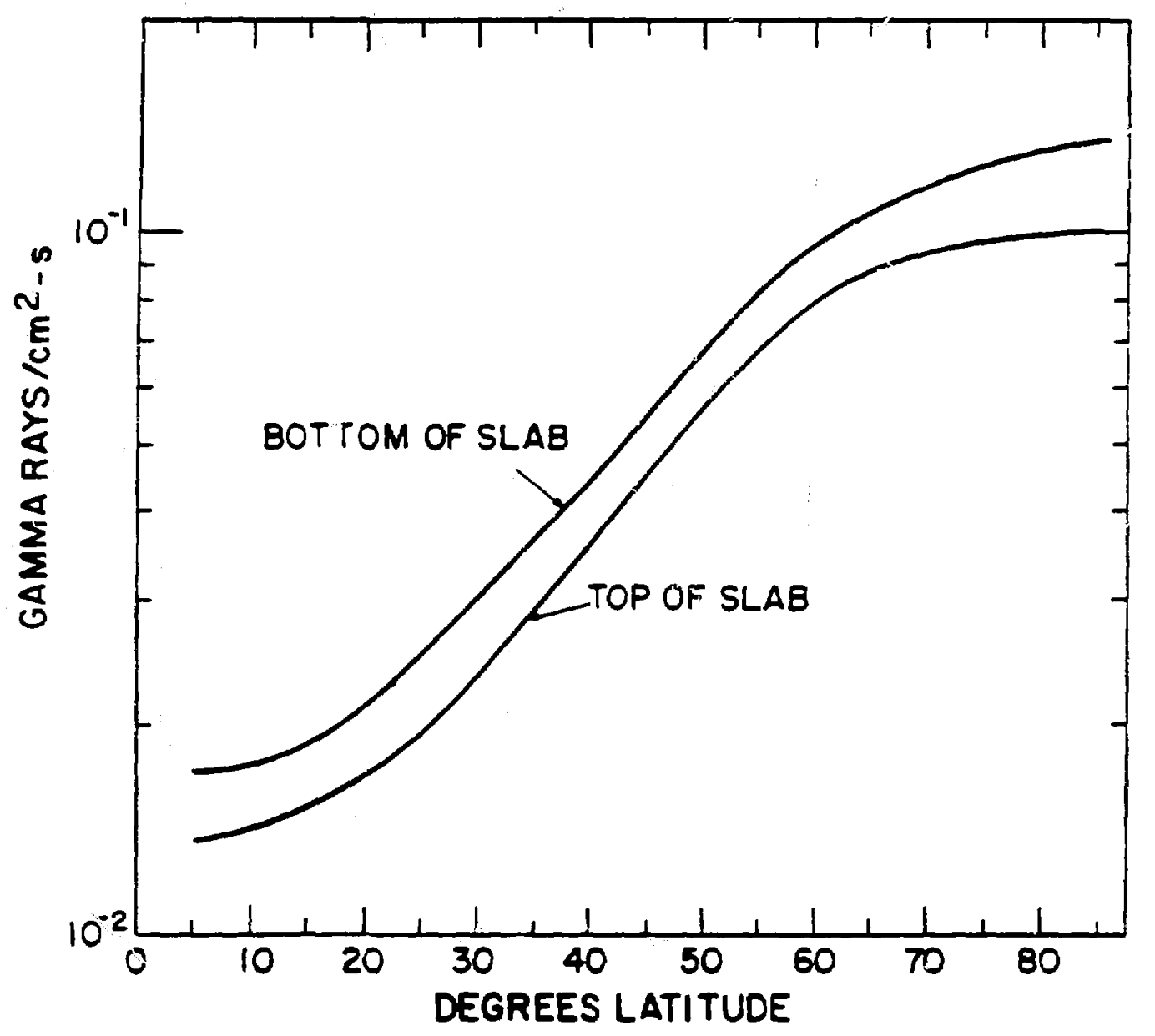

Fig. 4. Garma-ray leakages from satellite due to primary cosmic-ray protons as a function of latitude. Average solar activity. 


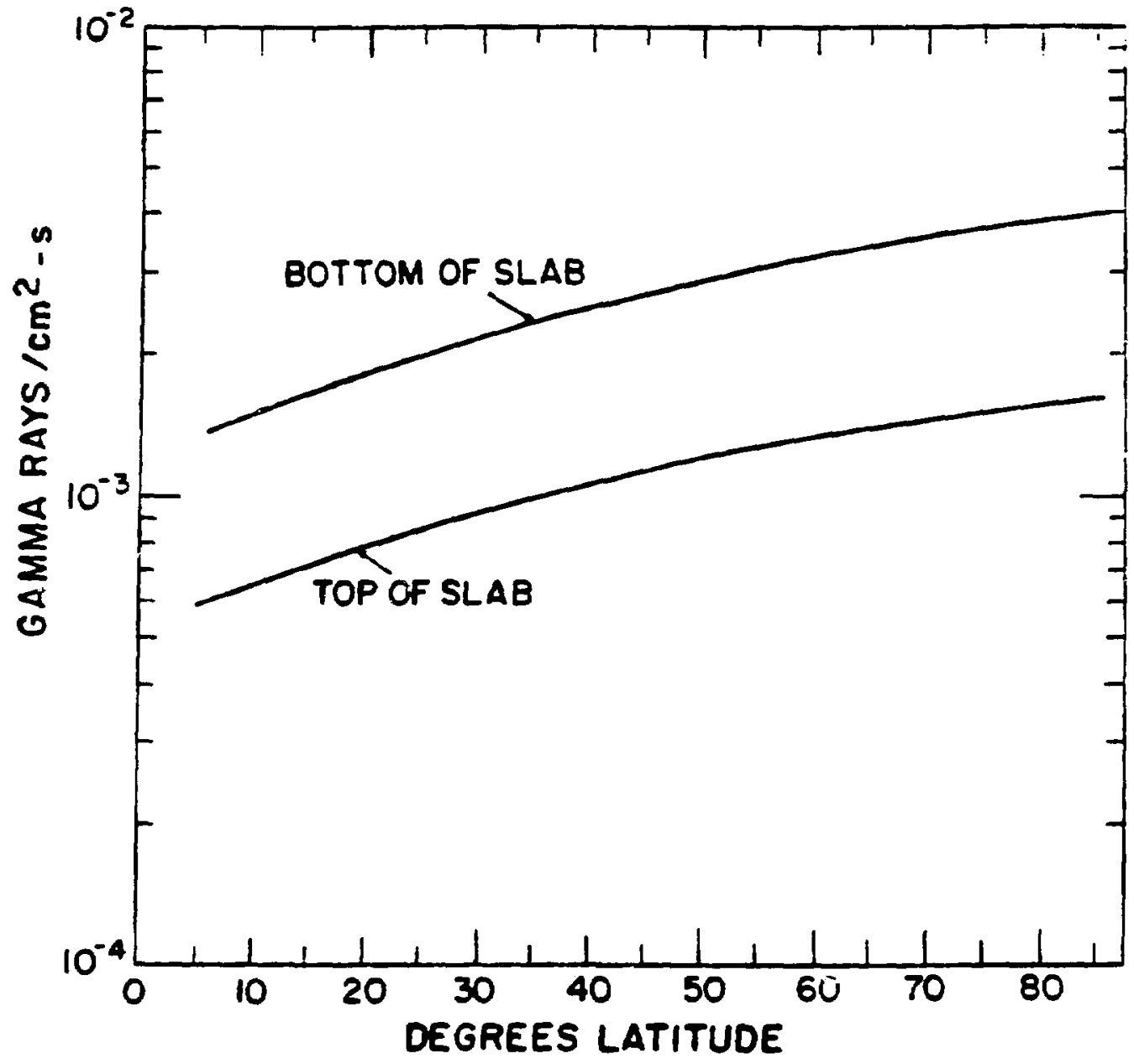

Fig. 5. Gamma-ray leakages from satellite due to primary cosmic-ray neutrons as a function of latitude. Average solar activity. 
GAMMA.AAY LEAKAGE SPECTRUM PROTON AND NEUTRON COMPONENTS OF PRIMARY COSMIC.RAY NUCLEON FLUX INCIDENT ON $3.3 \mathrm{~g} / \mathrm{cm} \mathrm{m}^{2}$ OF ALIJMINUMA LATITUDE = 35 DEGREES. AVERAGE SOLAR ACTIVITY.

GAMMA.RAY ENERGY

\begin{tabular}{c} 
(MeV) \\
\hline Above 100 \\
$10-100$ \\
$4-10$ \\
$2-4$ \\
$0.5-2$ \\
$0.1-0.5$ \\
$0.01-0.1$
\end{tabular}

\begin{tabular}{|c|c|c|c|}
\hline \multicolumn{2}{|c|}{ PROTONS } & \multicolumn{2}{|c|}{ NEUTRONS } \\
\hline TOP & BOTTOM & TOP & BOTTOA \\
\hline 0.5 & 3.8 & 1.8 & 9.8 \\
\hline 12.2 & 6.7 & 7.4 & 24.4 \\
\hline 12.3 & 5.2 & 7.3 & 24.8 \\
\hline 5.0 & 6.1 & 8.5 & 6.1 \\
\hline 15.6 & 22.8 & 43.3 & 18.6 \\
\hline 32.2 & 37.8 & 20.2 & 10.1 \\
\hline 22.2 & 17.6 & 11.5 & 6.2 \\
\hline
\end{tabular}

VII. COSMIC-RAY INDUCED NEUTRON AND GAMMA-RAY LEAKAGE AND SPECTRA FROM SURFACE OF A SFACE STATION AS A FUNCTION OF LATITUDE; AVERAGE SOLAR ACTIVITY

These calculations parallel those made for a satellite (Section $V$ ) and will therefore not be discussed in detail here. The geometry assumed for the space station was again a sphere, only this time the radius was increased to $5.72 \mathrm{~m}$ and the density $(\rho)$ to $0.173 \mathrm{~g} / \mathrm{cm}^{3}$. This gives a $\rho R$ of $99 \mathrm{~g} / \mathrm{cm}^{2}$ and a weight of $1.4 \times 10^{5} \mathrm{~kg}\left(3 \times 10^{5} \mathrm{ibs}\right)$. The neutron and gamma-ray leakages are plotted in $\mathrm{Fig} .6$ and summarized in Table VII. Compared to the leakages from a satellite (Fig. 3), the space station values are higher by 2 to 3 orders of magnitude for the neutrons and gamma-rays, respectively. The spectra of the leakage neutrons and photons are displayed in Table VIII. Again, compared to the satellite case (Table V), the spectra for the space station are much softer. Qualitatively, this is because of the larger thickness and density of the space station. Hence, neutrons and photons suffer more collisions and degradation in energy, in traveling to the surface.

VIII. COSMIC-RAY INDUCED WHOLE-BODY DOSE TO ASTRONAUT AS A FUNCTION OF LATITUDE; AVERAGE SOLAR ACTIVITY

These calculations to determine the whole-body dose equivalent rate to an astronaut were made with the original numerical HASL/KO'B cascade code (Appendix A) assuming a $30-\mathrm{cm}$ thick tissue phantom and using depth-dose curves for the particles of interest. Normal solar activity conditions (Ehmert potential $=200 \mathrm{MV}$ ) were stipulated. A curve of dose equivalent rate $(\mathrm{mrem} / \mathrm{h})$ versus latitude is shown in Fig. 7. The strong dependence on latitude is again observed, about a factor of 15 in going from 0 to 90 degrees. The time required to receive a dose 


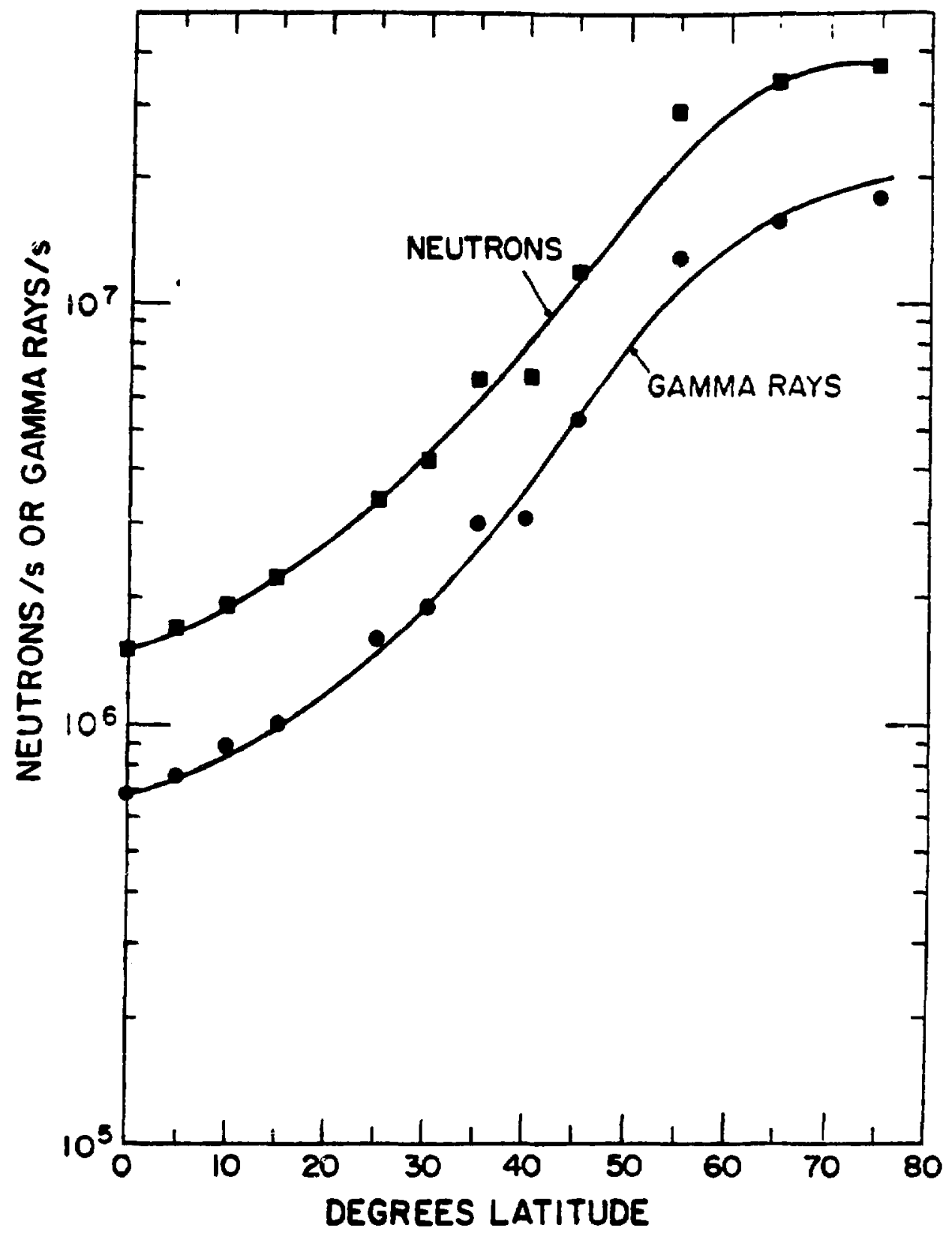

Fig. 6. Cosmic-ray induced neutron and gamma-ray leakages from surface of space station as a function of latitude. Average solar activity. 
TABLE VII

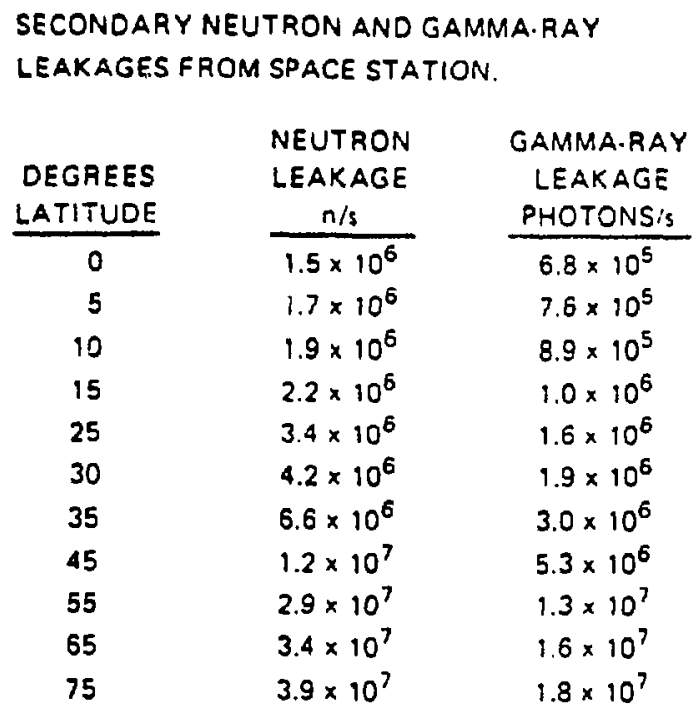

of 100 mrem varies from 50 to 700 hours. A rough estimate of the contribution of leakage particles from the top of the atmosphere to the latter dose rate was made and found to be negligible - about a factor of 5 less.

\section{CONCLUSIONS}

In view of the large amount of calculational results and the different uses to which these data might put, it is difficult to present a general summary that would be useful to all of the workers in this field. If, for example, one is interested in the detection problem, the magnitude of the leakage currents is not sufficient by itself unless the spectrum is known. The latter information is very detailed in nature and would require going back to a computer printout to retrieve the data. Hence, we will try to place the results in some sort of perspective by listing the magnitudes of the various components at two selected latitudes - 35 and 65 degrees. This is shown in Table IX. Without trying to indicate all of the trends evident in the table, we see first the very pronounced influence of latitude variations. The much larger leakage from a space station as compared to a satellite is also vividly illustrated; note that both these objects were approximated with idealized geometries. As for whole-body doses (Fig. 7), the factor of 15 difference at extremes of latitude is very important in determining how long one should perform tasks in outer space. 


\section{TABLE VIII}

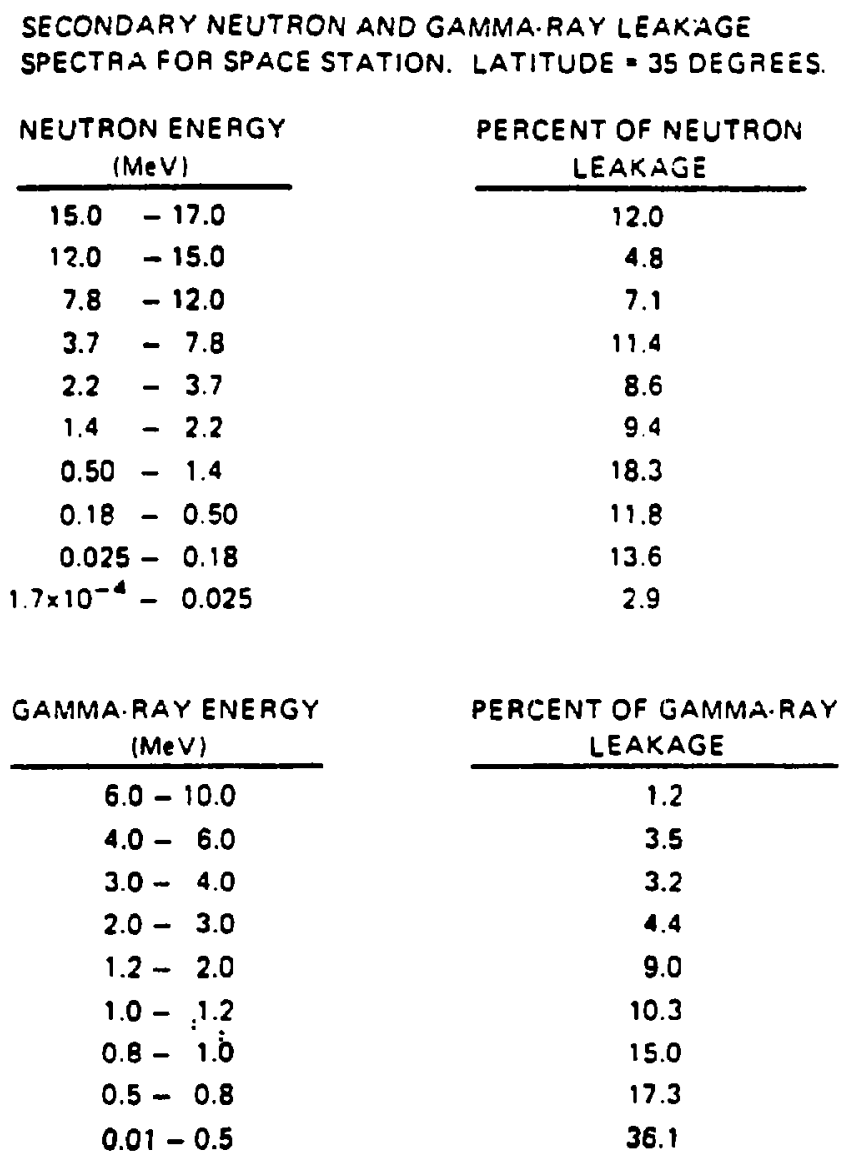




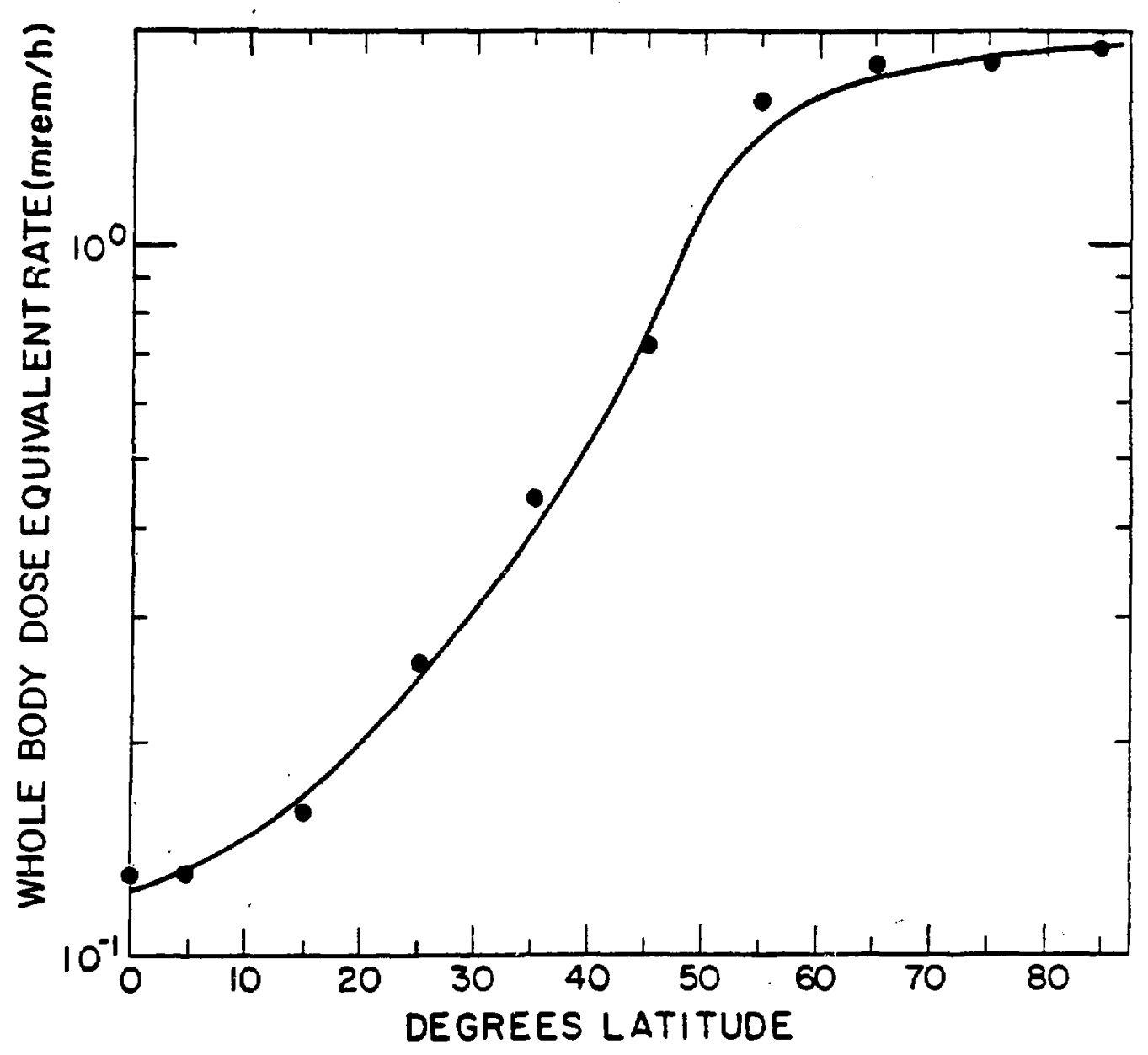

Fig. 7. Whole body dose equivalent rates to astronaut, from cosmic radiation, as a function of latitude. Average solar activity. 
TABLE IX

NEUTRON AND GAMMA-RAY LEAKAGES CALCULATED FOR LOW-ORBIT SPACE APPLICATIONS

AT 35 AND 65 DEGRIES LATITUDE. EHMERT POTENTIAL = 200 MV (AVERAGE SOLAR ACTIVITY).

35-Degree Latitude

Neutrons Photons

$0.66 / \mathrm{cm}^{2}-\mathrm{s}$

$0.63 / \mathrm{cm}^{2}-\mathrm{s}$

$0.11 / \mathrm{cm}^{2}-5$

$1.1 / \mathrm{cm}^{2}-5$

Diffuse cosmic gamma radiation

Leakage from satellite

(a) Cosmic-ray induced neutrons

(b) Direct production

Leakage from space station

Cosmic-ray induced neutrons
65-Degree Latitude

Neutrons Photons
$2.3 \times 10^{4} / \mathrm{s} \quad 1.6 \times 10^{3} / \mathrm{s}$
$1.3 \times 10^{5} / \mathrm{s}$
$9.3 \times 10^{3} / \mathrm{s}$
$0.051 / \mathrm{cm}^{2}-\mathrm{s}$
$0.0035 / \mathrm{cm}^{2}-\mathrm{s}$
$0.29 / \mathrm{cm}^{2}-5$
$0.021 / \mathrm{cm}^{2}-\mathrm{s}$
$0.03 / \mathrm{cm}^{2}-\mathrm{s}$
$0.1 / \mathrm{cm}^{2}-\mathrm{s}$

$6.6 \times 10^{6} / \mathrm{s}$

$3.0 \times 10^{6} / \mathrm{s}$

$0.73 / \mathrm{cm}^{2}-5$
$3.4 \times 10^{7} / 5$

$8.3 / \mathrm{cm}^{2}-5$
$1.6 \times 10^{7} / \mathrm{s}$

$3.8 / \mathrm{cm}^{2}-5$ 


\section{APPENDIX A \\ THE HASL/KO'B ${ }^{*}$ COSMIC RAY INTERACTION CASCADE CODE}

Around 1909, scientists generally believed that the ionizing radiation was entirely of terrestrial origin, due either to radioactive minerals in the earth or traces of the gaseous emanations arising from such minerals, or both. In 1909 and 1910, A Gockel, 5 a Swiss scientist, studied the phenomenon at high altitudes during balloon ascents. If the ionizing radiation had come from the earth, its intensity should have diminished at great heights because passage through a considerable layer of air would involve absorption and consequent attenuation. Results indicated that ionization intensity did actually decrease at first but then started to increase at higher altitude. At $4500 \mathrm{~m}$, Gockel found that an electroscope was discharged even more rapidly than at the earth's surface. From further experiments by others around 1913, it was concluded that the rays were of cosmic origin; and they were referred to as "Höhenstrahlung, "which might be translated as "high-altitude rays." Although it is common to speak of cosmic "ays as though they involve a single type of radiation, they are actually very $\mathrm{co}^{\circ} \mathrm{slex}$. The primary rays in outer space are relatively simple, but interaction, with matter changes their nature as they pass through the atmosphere.

The primary cosmic-ray flux in the solar system at the earth's orbit is a mixture of energetic protons, $\alpha$ particles, and a small admixture of heavier nuclei. This flux and its composition vary over the 11-year solar activity cycle.

It has been shown theoretically ${ }^{6}$ that the effect on the galactic cosmic-ray spectrum of passage through the interplanetary medium is about the same as wolid be produced by a heliocentric electrical potential with a magnitude at the earth's orbit equal to the energy lost by interacting with the solar wind. The approximation at cosmic-ray energies above about $50 \mathrm{MeV}$ appears to be a good one and has been used by Ehmert in 1959,7 Freier and Waddington in 1966,8,9 and Cleghorn et 2.1. in 1971.10 The ground-level counting rates of several cosmic-ray neutron monitors have been related to the primary cosmic-ray spectrum by means of the Ehmert potentials. "T The incident cosmic-ray nuclear spectrum at the earth's orbit can thus be computed from a knowledge of the appropriate ground level neutron-monitor counting rate over the course of the solar activity cycle.

The earth's geomagnetic field deflects cosmic-rays incident on the earth's orbit depending on their rigidity and angle of incidence. Each angle of incidence involves a critical rigidity below which the incoming particle cannot interact with the atmosphere. The cutoff field was calculated using ANGRI.12

After passing through the interplanetary medium and the geomagnetic field, the primary cosmic rays propagate by means of the nucleonic cascade through the atmosphere. The principal interactions produced by cosmic-rays in the atmosphere are

HASL, formerly the Health and Safety Laboratory of the AEC in New York, has been changed to the Department of Energy (DOE) Environmental Measurement Laboratory. Keran $O^{\prime} B r i e n\left(K O^{\prime} B\right.$ ) is the author of the Code. 


$$
\begin{aligned}
& p+\operatorname{air} \rightarrow p+n+\pi^{ \pm}+\pi^{0}+k^{ \pm}+k^{0} \\
& n+\operatorname{air} \rightarrow p+n+\pi^{ \pm}+\pi^{0}+k^{ \pm}+k^{0} \\
& k^{ \pm} \rightarrow \mu^{ \pm}+\pi^{ \pm}+\pi^{0} \\
& k^{0} \rightarrow \pi^{ \pm}+\pi^{-}+\pi^{0} \\
& \pi^{ \pm} \rightarrow \mu+\nu . \\
& \pi^{0} \rightarrow 2 \gamma \rightarrow \text { electromagnetic showers. } \\
& \mu \rightarrow e+2 \nu \rightarrow \text { electromagnetic showers. }
\end{aligned}
$$

The principal branches of the cosmic-ray atmospheric propagation process are shown in Fig. A-1. Because the kaon contribution is not large and the various decay schemes are complicated, we have not diagrammed the kaon branches of the hadronic cascade.

The behavior of cosmic-rays in the earths's atmosphere, averaged over time, is governed by the stationary form of the Boltzmann equation. Having incorporated the relevant cross sections, stopping powers, and reactions into the Boltzmann equation, we have written two codes to solve it in two different ways, each with its particular advantages and corresponding disadvantages.

The first code LUIN (for linear, uniform, internuclear, nucleonic) is a straight-ahead code based on the work of Passow. 13 It exploits the fact that the transverse momentum of the emitted particles in the reactions noted above are confined to small values. LUIN allows for the production of kaons but does not transport them.

As a numerical straight-ahead code, LUIN is well adapted to deep penetration problems and to collision-density (or star-production) calculations. Since the straight-ahead form of the Boltzmann equation vanishes on a free surface, it will not yield a finite albedo and, in addition, it can treat electromagnetic cascade propagation only primitively.

The second code, CASHEL (for cascades, hadronic and electromagnetic) is a Monte Carlo code utilizing the same basic physical data as LUIN. It also takes into account kaon production and transport and allows, as well, the rare mesonnucleus collision. CASHEL utilizes the well-known CKP formula to give the angular distribution of emitted hadrons. It also transports the electrons and photons resulting from meson or muon decay. Thus CASHEL yields a finite albedo and treats electromagnetic shower propagation accurately. It is, however, quite slow, due to the length and complexity of the cascade chain (see Fig. A-1).

Finally, the electromagnetic shower-component of CASHEL is a Monte Carlo routine that takes into account the photoelectric effect, Compton scattering, pair production, bremsstrahlung, multiple scattering, ionization, and positron annihilation. 14 


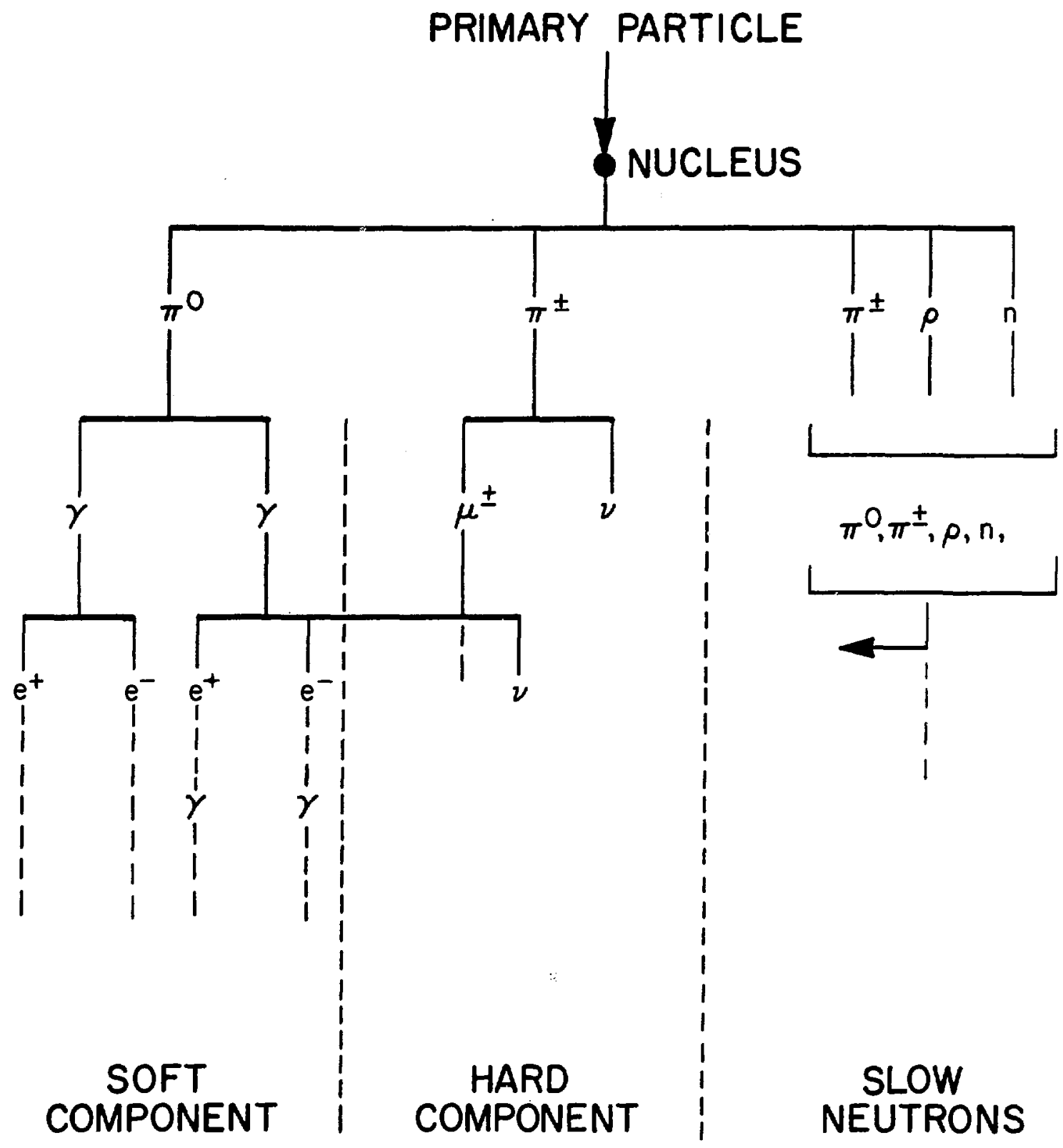

Fig. A-1. Development of soft and hard (secondary) components of cosmic rays. 


\section{DISCRETE ORDINATES CALCULATIONS}

The calculational results shown in Sections II, IV, and $V$ were obtained using the ONETRAN code. 15 ONETRAN is a discrete ordinates finite element code that solves the orie-dimensional multigroup transport equation in plane (slab), cylindrical, spherical, and two-angle plane geometries. Our calculations were performed using the $\mathrm{S}_{8} \mathrm{P}_{3}$ option $\left(\mathrm{S}_{8}\right.$ refers to the angular quadrature order and $\mathrm{P}_{3}$ is the order of the Legendre polynomial expansion used to represent the scattering anisotropy).

The starting point for the calculations was the specification of the source distribution, both as to intensity (spatially dependent) and spectra, in the system. For our problems, the source was expressed in terms of stars/g-s versus $\mathrm{g} / \mathrm{cm}^{2}$ of the medium. Multiplication by the appropriate density converts the source to stars $/ \mathrm{cm}^{3}-\mathrm{s}$. The product of the latter term and the neutrons $/ \mathrm{star}$ then gives neutrons $/ \mathrm{cm}^{3}-\mathrm{s}$. Thus, we have the source density at each spatial interval and for each of the neutron groups in the cross-section set. This source data is used by the code to calculate groupwise fluxes as a function of position and leakages at the system boundaries. Because our cross-section library contains information for neutrons and neutron-induced gamma rays, the code gives fluxes and leakages for both neutrons and gamma rays.

A multigroup, coupled ( 30 neutron by 12 photon), cross-section set for air and AT, Dased on ENDF/B-IV data, was generated by the Nuclear Data Group at LoS Alamos National Laboratory. The neutron energy group structure was the same as that given in Table 3 of Reference 1. In Table B-I is shown the gamma-ray group structure recommended by the Air Force and used for these studies.

TABLE B-I

PHOTON ENERGY GROUP STRUCTURE FOR DISCRETE ORDINATES CALCULATIONS

$$
E_{\max }=10 \mathrm{MeV}
$$

\begin{tabular}{r} 
Group \\
\hline 1 \\
2 \\
3 \\
4 \\
5 \\
6 \\
7 \\
8 \\
9 \\
10 \\
11 \\
12
\end{tabular}

Lower Boundary (MeV)

8.0

6.0

5.0

4.0

3.0

2.0

1.2

1.0

0.8

0.5

0.325

0.01 


\section{APPENDIX C}

\section{COSMIC-RAY INDUCED NEUTRON AND GAMMA-RAY FLUXES IN ATMOSPHERE}

In Section II, calculational results for neutron and gamma-ray leakages from the top of the atriosphere were presented. For these leakage calculations, made with the ONETRAN code, only the top $240 \mathrm{~g} / \mathrm{cm}^{2}$ of the atmosphere was considered; particles at greater depths did not contribute significantly to the top leakage. To compute the flux distribution in the atmosphere, a depth of 1100 $\mathrm{g} / \mathrm{cm}^{2}$ was specified - 220 mosh intervals, each $5 \mathrm{~g} / \mathrm{cm}^{2}$ thick. The $1100 \mathrm{~g} / \mathrm{cm}^{2}$ atmosphere was selected so as to avoid an air/vacuum interface at sea level $\left(1033 \mathrm{~g} / \mathrm{cm}^{2}\right)$. The source function, however, was set to zero for depths greater than the latter value.

Calculations were performed for Ehmert potentials of 200 and $1000 \mathrm{MV}$ and latitudes of $30-40^{\circ}$ and $50-60^{\circ}$. Results of the computations are displayed in Figs. $\mathrm{C}-1$ and $\mathrm{C}-2$. The general trends are the same as observed previously, namely, an increase in intensity with decreasing Ehmert potential and increasing latitude. Our calculated results are in fair agreement with those reported by Armstrong, Chandler, and Barish ${ }_{3} 6^{2}$ At $42^{\circ}$ latitude and solar minimum, they calculate a maximum flux of $5 \mathrm{n} / \mathrm{cm}^{2}-\mathrm{s}$ in the thermal to $20-\mathrm{MeV}$ energy range. From their curve of neutron flux versus depth, it is estimated that the flux maximum occurs at about $100 \mathrm{~g} / \mathrm{cm}^{2}$. Qur maximum flux for $30-40^{\circ}$ latitude and $200-\mathrm{MV}$ Ehmert potential is $3.3 \mathrm{n} / \mathrm{cm}^{2}-\mathrm{s}$ and occurs at $140 \mathrm{~g} / \mathrm{cm}^{2}$. At $800 \mathrm{~g} / \mathrm{cm}^{2}$, Armstrong et al. quote a flux of 0.10 as compared to our value of $0.15 \mathrm{n} / \mathrm{cm}^{2}-\mathrm{s}$. 


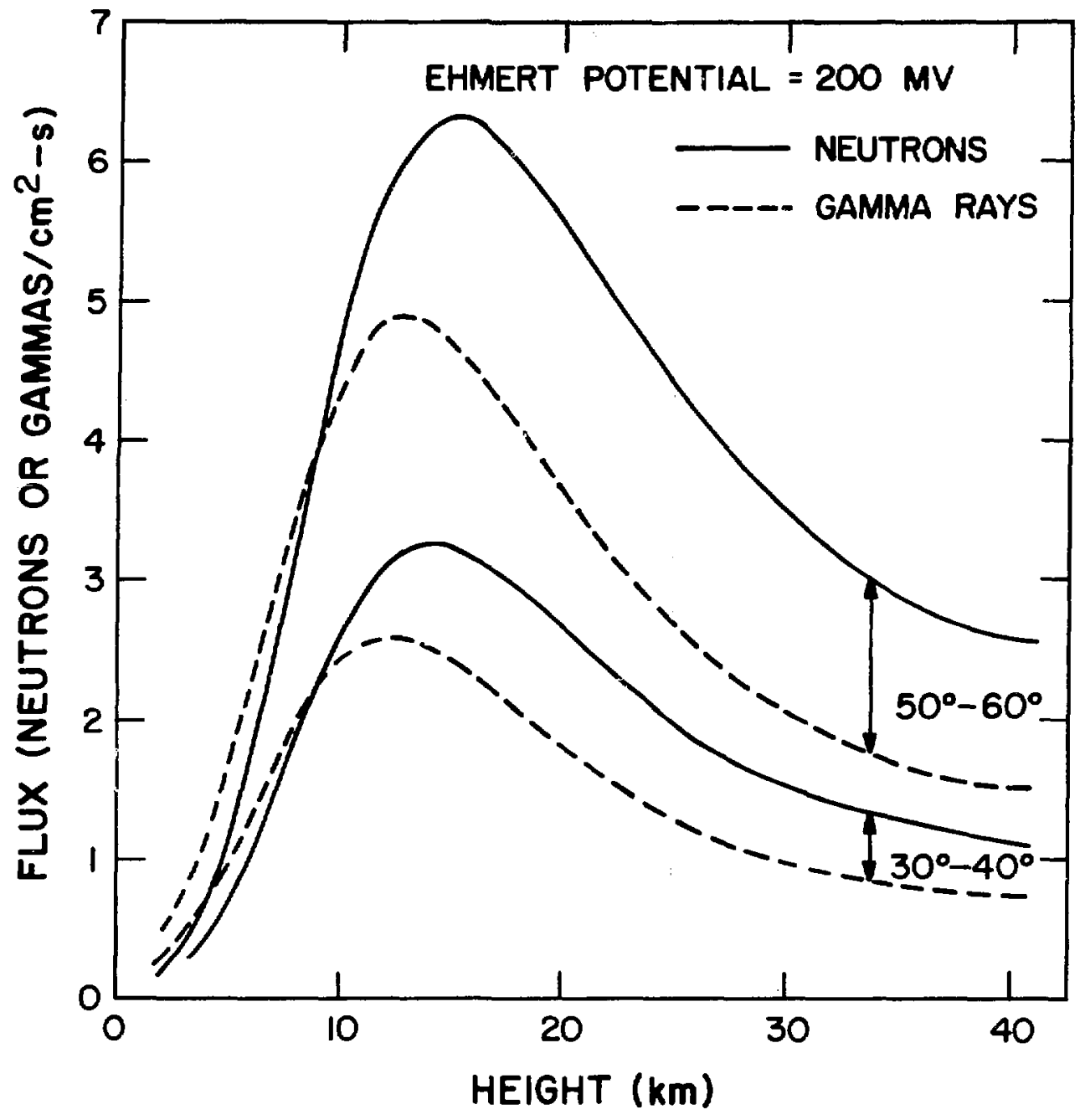

Fig. C-1. Neutron and gamma-ray fluxes in atmosphere due to cosmic-ray induced neutrons. Ehmert potential $=200 \mathrm{MV}$. Latitude $=30-40^{\circ}$ and $50-60^{\circ}$. 


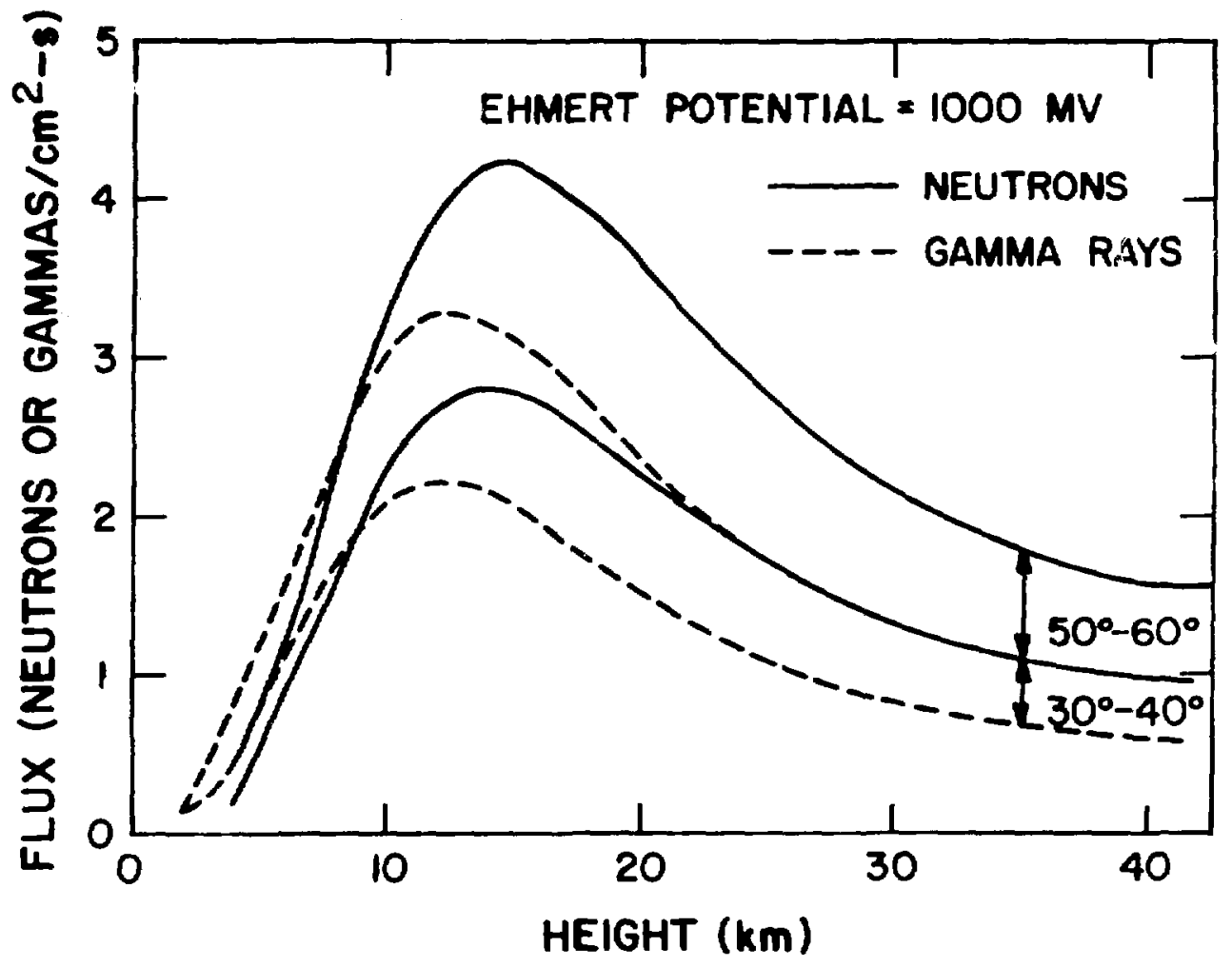

Fig. C-2. Neutron and gamma-ray fluxes in atmosphere due to cosmic-ray induced neutrons. Ehmert potential $=1000 \mathrm{MV}$. Latitude $=30-40^{\circ}$ and $50-60^{\circ}$. 


\section{REFERENCES}

1. K. O'Brien, H. A. Sandmeier, G. E. Hansen, and J. E. Campbell, "Cosmic Ray Induced Neutron Background Sources and Fluxes for Geometries of Air Over Water, Ground, Iron, and Aluminum," Journal of Geophysical Research 83, 114 (January 1,1978 ).

2. H. A. Sandmeier, G. E. Hansen, and K. O'Brien, unpublished data, 1979.

3. V. Schonfelder, U. Graser, and J. Daugherty, "Diffuse Cosmic and Atmospheric MeV Gamma Radiation from Balloon Observations," Ap. J. 217, 306 (1977).

4. Robert J. Gould, "Origin of Cosmic Rays," Am. J. Phys. 35, 376 (1967).

5. Samuel Glasstone, Sourcebook on Atomic Energy (US Atomic Energy Commission -- Division of Technical Information, D. Van Nostrand Co., Inc., 1967).

6. L. J. Gleeson and W. I. Axford, "Cosmic Rays in the Interplanetary Medium," Ap. J. 149, L 116 (1967).

7. A. Ehmert, "On the Modulation of the Primary Spectrum of Cosmic Rays from Solar Activity," Proc. Int. Conf. Cosmic Rays 4, Moscow, 1959, 140 (1959).

8. P. S. Freier and C. J. Waddington, "Modulation of Cosmic Rays by an Electric Field," Proc. Int. Conf. Cosmic Rays 9th I, London, 1966, 313 (1966).

9. P. S. Freier and C. J. Waddington, "The Helium Nuclei of the Primary Cosmic Radiation as Studied Over a Solar Cycle of Activity Interpreted in Terms of Electric Field Modulation," Space Science Rev. 4, 313 (1966).

10. T. F. Cleghorn, P. S. Freier, and C. J. Waddington, "The Modulation and Energy Spectrum of Highly Charged Cosmic Rays," Astrophysics Space Science 14, 424 (1971).

11. K. O'Brien and G. de P. Burke, "Calculated Cosmic Ray Neutron Monitor Response to Solar Modulation of Galactic Cosmic Rays," J. Geophys. Res. 78, 3013 (1973).

12. C. J. Bland and G. Cioni, "Geomagnetic Cutoff Rigidities in Nonvertical Directions," Earth Planet Sci. Lett. 4, 339 (1968).

13. C. Passow, "Phenomenologische Theorie zur Berechnung einer Kaskade aus schweren Te!!chen (Nukleonkaskade) in der Materie," Notiz A 285, Deut. Electron. Synchrotron, Hamburg, 1962.

14. K. O'Brien, "Electron Dosimetry Using Monte Carlo Techniques," in Computer Techniques in Radiation Transport and Dosimetry, M. P. Melson and T. M. Jenkins, Eds. (PTeniun Press, New York, 1980), pp 223-237. 
15. T. R. Hill, "ONETRAN: A Discrete Ordinates Finite Element Code for the Solution of the One-Dimensional Multigroup Transport Equation," Los Alamos Scientific Laboratory report LA-5990-MS (June 1975).

16. T. W. Armstrong, K. C. Chandler, and J. Barish, "Calculations of Neutron Flux Spectra Induced in the Earth's Atmosphere by Galactic Cosmic Rays," J. Geophys. Res. 78, 2715 (1973). 